DESY 06-075

May 2006

\title{
Detecting metastable staus and gravitinos at the ILC
}

\author{
H.-U. Martyn \\ 1. Physikalisches Institut, RWTH Aachen, Aachen, Germany and \\ Deutsches Elektronen-Synchrotron DESY, Hamburg, Germany
}

\begin{abstract}
A study of various SUSY scenarios is presented in which the lightest supersymmetric particle is the gravitino $\tilde{G}$ and the next-to-lightest supersymmetric particle is a scalar tau $\tilde{\tau}$ with lifetimes ranging from seconds to years. Gravitinos are interesting dark matter candidates which can be produced in decays of heavier sparticles at the International Linear Collider (ILC), but remain undetected in direct searches of astrophysical experiments. We investigate the detection and measurement of metastable staus, which may be copiously produced at the ILC either directly or via cascade decays. A proper choice of the experimental conditions will allow one to stop large samples of $\tilde{\tau}^{\prime} s$ in the calorimeters of the ILC detector and to study the subsequent decays $\tilde{\tau} \rightarrow \tau \tilde{G}$. Detailed simulations show that the properties of the stau and the gravitino, such as $\tilde{\tau}$ mass and lifetime and $\tilde{G}$ mass, can be accurately determined at a future ILC and may provide direct access to the gravitational coupling, respectively Planck scale.
\end{abstract}

\section{Introduction}

Supersymmetry (SUSY) provides a very attractive scenario to account for the amount of dark matter in the universe. If $R$-parity is conserved, the lightest supersymmetric particle (LSP) is stable and thus an ideal dark matter candidate. Most frequently discussed is a neutralino LSP. Another interesting candidate is the spin $3 / 2$ gravitino $\tilde{G}$, cf. ref. [1], which is part of the supersymmetric particle spectrum in extensions to supergravity, or local Susy. The mass of the gravitino is set by the Susy breaking scale $F$

$$
m_{3 / 2}=m_{\tilde{G}}=\frac{F}{\sqrt{3} M_{P}},
$$

where $M_{P}=\left(8 \pi G_{N}\right)^{-1 / 2} \simeq 2.4 \cdot 10^{18} \mathrm{GeV}$ is the reduced Planck scale and $G_{N}$ is Newton's constant. The masses of the other superparticles depend on the supersymmetry breaking mechanism and on the parameters of a specific model. In general $m_{3 / 2}$ is a free parameter in the theory and may extend over a wide range of $\mathcal{O}(\mathrm{eV}-\mathrm{TeV})$.

If the gravitino is indeed the LSP, it may be produced in cascade decays of heavy SusY particles. To be specific, the next-to-lightest supersymmetric particle (NLSP) is assumed to be a charged slepton, the scalar tau $\tilde{\tau}$. The dominant decay will then be $\tilde{\tau} \rightarrow \tau \tilde{G}$ and since the coupling is gravitational, the lifetime may be very long, ranging from seconds to years. The decay-width $\Gamma_{\tilde{\tau}}$ of the metastable $\tilde{\tau}$ NLSP is given by

$$
\Gamma_{\tilde{\tau} \rightarrow \tau \tilde{G}}=\frac{1}{48 \pi M_{P}^{2}} \frac{m_{\tilde{\tau}}^{5}}{m_{\tilde{G}}^{2}}\left[1-\frac{m_{\tilde{G}}^{2}}{m_{\tilde{\tau}}^{2}}\right]^{4} .
$$

This formula will be the basis of the present study. The $\tilde{\tau}$ decay rate, respectively lifetime $t_{\tilde{\tau}}=\Gamma_{\tilde{\tau}}^{-1}$, depends only on the masses $m_{\tilde{\tau}}$ and $m_{\tilde{G}}$ as well as on the Planck scale $M_{P}$ - no further supersymmetry parameters are required. Conversely, the 'supergravity Planck scale' can be expressed in terms of observable quantities

$$
M_{P}=\sqrt{\frac{t_{\tilde{\tau}} m_{\tilde{\tau}}}{48 \pi}} \frac{m_{\tilde{\tau}}^{2}}{m_{\tilde{G}}}\left[1-\frac{m_{\tilde{G}}^{2}}{m_{\tilde{\tau}}^{2}}\right]^{2} .
$$

A comparison of such a measurement at a particle collider experiment with the value determined in macroscopic gravitational experiments, $M_{P}=2.436(2) \cdot 10^{18} \mathrm{GeV}$ [2], would be a unique test of supergravity [3]. If the 
gravitational coupling of the $\tilde{\tau}$ decay is assumed, the direct measurement of the gravitino mass may be replaced by a more accurate determination from the decay-width. Rewriting eq. (2), the $\tilde{\tau}$ lifetime as a function of the stau and gravitino masses can be expressed to a good approximation as

$$
t_{\tilde{\tau}} \simeq 3.6 \cdot 10^{7} \mathrm{~s}\left[\frac{100 \mathrm{GeV}}{m_{\tilde{\tau}}-m_{\tilde{G}}}\right]^{4}\left[\frac{m_{\tilde{G}}}{100 \mathrm{GeV}}\right] .
$$

The cosmological production of gravitino dark matter proceeds essentially via two mechanisms: thermal production in 2-body QCD interactions, e.g. $g g \rightarrow \tilde{g} \tilde{G}$ or $q \tilde{g} \rightarrow q \tilde{G}$, in the reheating phase following inflation [4]; and/or late decays of the next-to-lightest sparticle, usually a slepton or neutralino, which freezes out as usual and decays only after the big bang nucleosynthesis phase, the prediction of which should not be destroyed by the hadronic decays [5]. Comparing the calculated gravitino relic density with the observed dark matter density puts constraints on the NLSP and gravitino masses and on the parameters for supersymmetric models. Collider experiments offer a unique possibility to detect metastable staus and to study the properties of gravitinos, which otherwise cannot be observed directly in astrophysical experiments. Some phenomenological investigations to detect long-lived staus and to measure their lifetime at the LHC and ILC are discussed in [6-8].

In the present study the excellent potential of future ILC experiments and detectors will be explored to observe and detect metastable $\tilde{\tau}^{\prime} s$, measure precisely their mass and lifetime, and to determine in an independent way the gravitino mass. In section 2 the investigated physics scenarios will be presented. Details on experimental aspects, detector requirements and event simulation will be given in sections 3 and 4 . The experimental analyses and case studies are described in section 5 . The results are discussed in section 6 , followed by conclusions.

\section{Physics scenarios}

Several SuSY benchmark models which have been recently discussed in the literature are chosen for the case studies. They represent different supersymmetry breaking mechanisms and a variety of stau and gravitino masses leading to different experimental conditions. In general, the models are consistent with cosmological constraints, as claimed by the authors. Here they should just serve as valid examples of 'typical' spectra. The gravitino masses are somewhat arbitrary. However, most important, the results can be easily transformed to other scenarios with different stau and gravitino masses. The masses of the sleptons, light neutralinos and charginos, relevant for the benchmark models under study, are compiled in table I.

Gauge mediated symmetry breaking (GMSB) usually occurs at rather low scales and a light gravitino is naturally the LSP [9]. Typical masses are of order $\mathrm{eV}$ to $\mathrm{keV}$ which may, however, be extended in the GeV range [10]. The GMSB scenario SPS 7 from the Snowmass points [11] is chosen as reference model. It is described by the conventional parameters $\Lambda=40 \mathrm{TeV}, M_{m}=80 \mathrm{TeV}, N_{m}=3, \tan \beta=15$ and $\operatorname{sign} \mu=+$, where $\Lambda$ is the universal soft Susy breaking scale, $M_{m}$ and $N_{m}$ are the messenger scale and index, $\tan \beta$ is the ratio of the vacuum expectation values of the two Higgs fields, and $\operatorname{sign} \mu$ is the sign of the higgsino mass parameter $\mu$. The gravitino mass is set arbitrarily to $m_{\tilde{G}}=0.1 \mathrm{GeV}$.

In supergravity mediated symmetry breaking (SUGRA) the gravitino mass $m_{3 / 2}$ is a free parameter and of the same order as the other sparticle masses [12]. If the NLSP is required to be the charged $\tilde{\tau}$, then the common scalar mass $m_{0}$ in minimal versions has to be small and much lower than the common gaugino mass $M_{1 / 2}$. In the scenario FS 600 of ref. [7] the mSUGRA parameters are $m_{0}=0 \mathrm{GeV}, M_{1 / 2}=600 \mathrm{GeV}, A_{0}=0 \mathrm{GeV}, \tan \beta=10$ and $\operatorname{sign} \mu=+$, where $A_{0}$ is the common trilinear coupling. The gravitino mass is chosen to be $m_{\tilde{G}}=50 \mathrm{GeV}$. The authors of the GDM series [8] require a tighter mSUGRA definition with unified scalar and gravitino masses $m_{3 / 2}=m_{0}$. The parameters of GDM $\epsilon$ are $m_{0}=m_{3 / 2}=20 \mathrm{GeV}, M_{1 / 2}=440 \mathrm{GeV}, A_{0}=25 \mathrm{GeV}, \tan \beta=15$ and $\operatorname{sign} \mu=+$. The model GDM $\zeta$ is given by $m_{0}=m_{3 / 2}=100 \mathrm{GeV}, M_{1 / 2}=1000 \mathrm{GeV}, A_{0}=127 \mathrm{GeV}$, $\tan \beta=21.5$ and $\operatorname{sign} \mu=+$. The spectrum of GDM $\eta$ differs from the previous one essentially only by a lighter gravitino, thus $m_{0}=m_{3 / 2}=20 \mathrm{GeV}, M_{1 / 2}=1000 \mathrm{GeV}, A_{0}=25 \mathrm{GeV}, \tan \beta=23.7$ and $\operatorname{sign} \mu=+$. 
Table I: Spectra of sleptons, light neutralinos and charginos [masses in GeV] in Susy scenarios with a gravitino LSP, masses of the first and second generation are identical; the gravitino masses $[\mathrm{GeV}]$ used in the case studies are given in the last line

\begin{tabular}{lccccccc}
\hline & 1 & 2 & 3 & 4 & 5 & 6 & 7 \\
$\tilde{\ell}, \tilde{\chi}, \tilde{G}$ & SPS 7 & FS 600 & GDM $\epsilon$ & GDM $\zeta$ & GDM $\eta$ & $\tilde{\chi}$ MSB P1 & $\tilde{\chi}$ MSB P3 \\
\hline$\tilde{\tau}_{1}$ & 123.4 & 219.3 & 157.6 & 340.2 & 322.1 & 185.2 & 102.5 \\
$\tilde{\tau}_{2}$ & 264.9 & 406.5 & 307.2 & 659.2 & 652.2 & 341.5 & 356.9 \\
$\tilde{\nu}_{\tau}$ & 249.6 & 396.4 & 290.9 & 649.5 & 641.5 & 327.7 & 346.9 \\
\hline$\tilde{e}_{R}$ & 130.9 & 227.2 & 175.1 & 381.4 & 368.5 & 192.9 & 109.5 \\
$\tilde{e}_{L}$ & 262.8 & 405.6 & 303.0 & 662.7 & 655.3 & 340.1 & 357.4 \\
$\tilde{\nu}_{e}$ & 250.1 & 397.6 & 292.8 & 658.1 & 650.7 & 328.0 & 247.4 \\
\hline$\tilde{\chi}_{1}^{0}$ & 163.7 & 243.0 & 179.4 & 426.5 & 426.5 & 203.6 & 189.2 \\
$\tilde{\chi}_{2}^{0}$ & 277.9 & 469.6 & 338.2 & 801.9 & 801.5 & 385.5 & 263.8 \\
$\tilde{\chi}_{1}^{ \pm}$ & 275.5 & 469.9 & 338.0 & 801.9 & 801.4 & 388.2 & 251.5 \\
\hline$\tilde{G}$ & 0.1 & 50 & 20 & 100 & 20 & 50 & 25 \\
\hline
\end{tabular}

In gaugino mediated symmetry breaking ( $\tilde{\chi} \mathrm{MSB})$ the gravitino may be the LSP with a mass comparable to the other sparticles [13]. In the scenarios proposed by [14] a gravitino LSP can be accompanied by a stau, sneutrino or neutralino NLSP, where the latter options are essentially unobservable. The spectra of the $\tilde{\tau}$ NLSP models are determined by the parameters $M_{1 / 2}=500 \mathrm{GeV}, \tan \beta=10$, sign $\mu=+$ and the soft Higgs masses, chosen as $m_{\tilde{h}_{1}}^{2}=m_{\tilde{h}_{2}}^{2}=0 \mathrm{TeV}^{2}$ for point $\tilde{\chi} \mathrm{MSB} \mathrm{P} 1$, and $m_{\tilde{h}_{1}}^{2}=0 \mathrm{TeV}^{2}, m_{\tilde{h}_{2}}^{2}=0.5 \mathrm{TeV}^{2}$ for point $\tilde{\chi} \mathrm{MSB} \mathrm{P} 3$. An interesting feature of this particular gaugino mediation scenario is that a naive dimensional analysis provides a lower bound on the gravitino mass of $m_{3 / 2} \gtrsim 10 \mathrm{GeV}$ [15]. In the present case studies values of $m_{\tilde{G}}=50 \mathrm{GeV}$ and $m_{\tilde{G}}=25 \mathrm{GeV}$ are chosen for $\mathrm{P} 1$ and $\mathrm{P} 3$, respectively.

The sparticle spectra of table I are calculated from the original set of parameters using the programs SPheno [16] for models 1, 2 and SuSpect [17] for models $3-7$.

\section{Stau detection \& measurement principles}

The programme to determine the properties of the metastable stau and to measure, independently, the mass of the gravitino consists of several parts:

- identify $\tilde{\tau}$ by the characteristic heavy ionisation $-d E / d x \propto 1 / \beta^{2}$ in the TPC;

- determine $\tilde{\tau}$ mass from two-body kinematics of $e^{+} e^{-} \rightarrow \tilde{\tau}_{1} \tilde{\tau}_{1}$ production;

- follow low momentum $\tilde{\tau}$ candidate until it stops inside detector, record $\tilde{\tau}$ location and time stamp $t_{0}$;

- trigger decay $\tilde{\tau} \rightarrow \tau \tilde{G}$ at $t_{\text {trig }}$ uncorrelated to beam collision;

- associate vertex to previously recorded stopping point, get $\tilde{\tau}$ lifetime $t_{\tilde{\tau}}=t_{\text {trig }}-t_{0}$;

- measure $\tau$ recoil spectra in calorimeter, get gravitino mass $m_{\tilde{G}}$.

The requirements on the detector performance and operation will be discussed in the following.

The detector is taken from the TESLA TDR [18]; a cross section through one quadrant is displayed in figure 1. This concept serves as a baseline for a future LDC detector [19]. In particular the calorimeter layout and depth may be taken as representative for various detector designs discussed in the ILC community. The main characteristics of the detector, relevant to the present study, are:

- A TPC with excellent tracking performance, i.e. momentum resolution $\delta\left(1 / p_{t}\right)<2 \cdot 10^{-4} \mathrm{GeV}^{-1}$ and $\mathrm{dE} / \mathrm{dx}$ resolution $<5 \%$. 


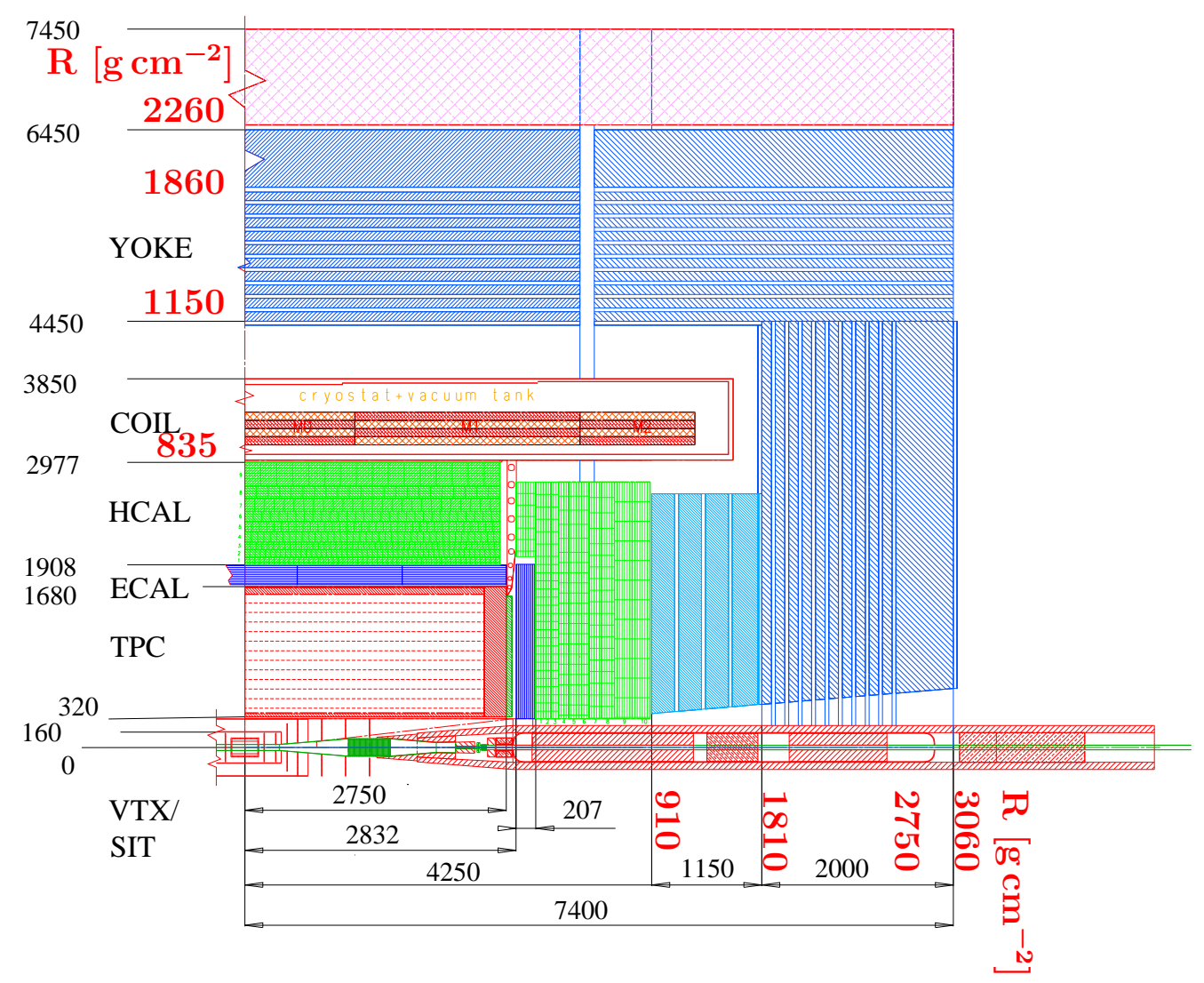

Figure 1: View of one quadrant of the TESLA detector [18], length units in $\mathrm{mm}$; the amount of material integrated perpendicular and along the beam direction is indicated by slightly larger (red) symbols as $R\left[\mathrm{~g} \mathrm{~cm}^{-2}\right]$

- A highly segmented hadronic calorimeter (HCAL), 40 samplings with $3 \times 3 \mathrm{~cm}^{2}$ lateral readout cells, leading to energy resolutions of $\delta E_{\text {had }}=0.5 \sqrt{E / \mathrm{GeV}} \oplus 0.04 E$ for hadrons and $\delta E_{\text {em }}=0.2 \sqrt{E / \mathrm{GeV}} \oplus 0.02 E$ for electromagnetic showers.

- An instrumented iron flux return yoke equipped with several layers for muon detection as well as for calorimetric measurements of hadron showers.

The amount of absorber material integrated along the radial and longitudinal directions, $R\left[\mathrm{~g} / \mathrm{cm}^{2}\right]$, can be read off the detector figure 1 . The range of a heavy, non-strongly interacting charged particle of mass $m$, is conveniently expressed by $R / m\left[\mathrm{~g} \mathrm{~cm}^{-2} \mathrm{GeV}^{-1}\right]$ and can be parametrised as a function of the scaled momentum $p / m=\beta \gamma$

$$
\log _{10}(R / m)=c_{1}+c_{2} \log _{10}(p / m)
$$

with $c_{1}=2.087$ and $c_{2}=3.227$ for steel [20]. Values of $\beta \gamma$ below which a heavy $\tilde{\tau}$ can be stopped in the hadron calorimeter or instrumented iron yoke depend on the location and angle of incidence. Typical ranges are listed in table II.

\section{Event generation}

Events are generated with the program PyтніA 6.3 [21] which includes initial and final state QED radiation as well as beamstrahlung [22]. The detector simulation is based on the detector proposed in the TESLA TDR [18] 
Table II: Acceptance ranges of maximal $\beta \gamma$ at which heavy $\tilde{\tau}^{\prime} s$ can be absorbed in the HCAL or instrumented iron yoke of the ILC detector

\begin{tabular}{ccc}
$m_{\tilde{\tau}}$ & $\beta \gamma($ HCAL $)$ & $\beta \gamma($ Yoke $)$ \\
\hline $125 \mathrm{GeV}$ & $0.41-0.46$ & $0.52-0.59$ \\
$250 \mathrm{GeV}$ & $0.33-0.37$ & $0.42-0.48$ \\
$375 \mathrm{GeV}$ & $0.29-0.33$ & $0.37-0.41$
\end{tabular}

and implemented in the Monte Carlo program Simdet 4.02 [23].

The analysis of SUSY scenarios with a gravitino LSP is quite different from conventional supersymmetric models. The metastable $\tilde{\tau}$ NLSP will be detected before it leaves or will be absorbed in the detector. Thus, there is a very clean signature without missing particles (except neutrinos from decays): the observed particle momenta are balanced but their moduli don't sum up to the cms energy

$$
\left|\sum_{i} \overrightarrow{p_{i}}\right| \simeq 0 \quad \text { and } \quad \sum_{i} p_{i}<\sqrt{s} .
$$

These features are very distinct from Standard Model background which can be efficiently rejected. They also allow the sparticle production and decay chains to be reconstructed from the complete event kinematics. Each SusY event contains two $\tilde{\tau}^{\prime} s$ which can be readily identified as highly ionising tracks and their passage through the detector can be accurately followed. The location of stopping $\tilde{\tau}^{\prime} s$ may be determined within a volume of a few $\mathrm{cm}^{3}$.

The decay $\tilde{\tau} \rightarrow \tau \tilde{G}$ is triggered by an isolated, high energy hadronic or electromagnetic cluster in the HCAL (threshold $\left.E_{h, e m}>10 \mathrm{GeV}\right)$, by a hadronic shower in the yoke $\left(E_{h}>10 \mathrm{GeV}\right)$ or by an energetic $\mu$ originating in the HCAL or yoke $\left(E_{\mu}>10 \mathrm{GeV}\right)$, occurring at any time not correlated to beam collisions. The main background is expected to come from cosmic rays and can be rejected by excluding decay vertices in the outermost detector layers or signals being initiated by muons from outside. Further excellent background discrimination is provided by requiring the decay vertex to coincide with a previously recorded $\tilde{\tau}$ stopping point. The $\tau$ decay modes contributing to the analysis are the leptonic 3-body decays $\tau \rightarrow \mu \nu_{\mu} \nu_{\tau}(17.4 \%)$ and $\tau \rightarrow e \nu_{e} \nu_{\tau}(17.8 \%)$, and the hadronic decays $\tau \rightarrow \pi \nu_{\tau}$ (11.1\%), $\tau \rightarrow \rho \nu_{\tau} \rightarrow \pi^{ \pm} \pi^{0} \nu_{\tau}$ (25.4\%) and $\tau \rightarrow 3 \pi \nu_{\tau} \rightarrow \pi^{ \pm} \pi^{+} \pi^{-} \nu_{\tau}+\pi^{ \pm} \pi^{0} \pi^{0} \nu_{\tau}$ $(19.4 \%)$.

The $\tau$ recoil energy gives access to the gravitino mass

$$
E_{\tau}=\frac{m_{\tilde{\tau}}}{2}\left(1-\frac{m_{\tilde{G}}^{2}}{m_{\tilde{\tau}}^{2}}\right) \quad \text { for } \quad m_{\tau}^{2} \ll m_{\tilde{\tau}}^{2}
$$

The sensitivity to low gravitino masses decreases rapidly, e.g. for $m_{\tilde{G}}=0.1 m_{\tilde{\tau}}$ and $m_{\tilde{\tau}}$ precisely known the energy $E_{\tau}$, which is also the maximum energy of the observable $\tau$ decay products, has to be measured with a precision well below one percent.

\section{Experimental analyses - case studies}

A big advantage of the ILC is that the centre of mass energy can be adjusted in order to control the sparticle production and the number of staus trapped in the detector. A range-out in the calorimeter is preferred for measuring the kinematics of $\tilde{\tau}$ decays and thus the gravitino mass, while all staus stopped somewhere in the detector can be used for determining the lifetime. Low momentum $\tilde{\tau}^{\prime} s$ with a suitable $\beta \gamma$ factor can be produced either directly or via cascade decays from light sleptons, e.g. $\tilde{e}_{R} \rightarrow e \tau \tilde{\tau}_{1}$, or neutralinos, $\tilde{\chi}_{1}^{0} \rightarrow \tau \tilde{\tau}_{1}$. However, all these processes - $\tilde{\tau}_{1} \tilde{\tau}_{1}, \tilde{e}_{R} \tilde{e}_{R}, \tilde{\mu}_{R} \tilde{\mu}_{R}$ and $\tilde{\chi}_{1}^{0} \tilde{\chi}_{1}^{0}$ - rise only slowly above kinematic threshold with cross sections $\sigma \propto \beta^{3}$, thus providing relatively low rates. Obviously, choosing $\tilde{\tau}_{1} \tilde{\tau}_{1}$ pair production as the only source 
is not optimal. A more efficient reaction, if kinematically accessible, is the associated selectron production $e^{+} e^{-} \rightarrow \tilde{e}_{R} \tilde{e}_{L} \rightarrow e \tau \tilde{\tau}_{1} e \tilde{\chi}_{1}^{0}$, which increases much faster as $\sigma \propto \beta$ near threshold [24]. Note, such a threshold behaviour also applies to $e^{-} e^{-} \rightarrow \tilde{e}_{R} \tilde{e}_{R}$ production [25].

For the present studies the collider energies are not always optimised. Instead, the often proposed 'standard' energies of $500 \mathrm{GeV}$ (models 2, 3, 7) and $800 \mathrm{GeV}$ (models 4, 5) are assumed, as well as some special settings around $420 \mathrm{GeV}$ (models 1, 6). The integrated luminosities are moderate and chosen such as to enable a reasonable measurement of the $\tilde{\tau}$ decay spectra. The results obtained may be easily scaled to apply to different running conditions, event statistics and detector efficiencies.

The experimental analysis and techniques will be discussed representatively in some detail for the GDM $\epsilon$ scenario. For the other benchmark models the main features and results will be presented in a more compact form. All results are summarised in table III.

\section{1. mSUGRA scenario GDM $\epsilon$}

The assumptions for the case study of the GDM $\epsilon$ scenario consist of the observables $m_{\tilde{\tau}}=157.6 \mathrm{GeV}$, $t_{\tilde{\tau}}=2.6 \cdot 10^{6} \mathrm{~s}, m_{\tilde{G}}=20 \mathrm{GeV}$ and the experimental conditions of $\sqrt{s}=500 \mathrm{GeV}$ centre of mass energy, $\mathcal{L}=100 \mathrm{fb}^{-1}$ integrated luminosity, and $\sigma_{S U S Y}=300 \mathrm{fb}$ as inclusive $\tilde{\tau}$ production cross section.

The scaled momentum distribution $\beta \gamma$ of $\tilde{\tau}^{\prime} s$ produced in various reactions is shown in figure $2 \mathrm{a}$. The majority of particles, coming from diagonal slepton and neutralino pairs and peaking around $\beta \gamma \simeq 1$, will leave the detector. One observes, however, a second peak at low $\beta \gamma \lesssim 0.5$ which stem from cascade decays of $\tilde{e}_{R} \tilde{e}_{L}$ production and which may be stopped inside the detector, see $\beta \gamma$ ranges in table II. One expects $N_{\tilde{\tau}}^{\text {hcal }}=4100$ and $N_{\tilde{\tau}}^{\text {yoke }}=1850 \tilde{\tau}^{\prime} s$ trapped in the calorimeter and yoke, respectively.

The stau mass measurement is based on the kinematics of prolific pair production $e^{+} e^{-} \rightarrow \tilde{\tau}_{1} \tilde{\tau}_{1}$, see magenta curve in figure $2 \mathrm{a}$, to be identified as a pair of collinear, non-interacting particles with momenta $p_{\tilde{\tau}}<\sqrt{s} / 2=E_{\tilde{\tau}}$, i.e. well below the beam energy. Determining the mean value of the $\tilde{\tau}$ momentum with an accuracy of $\left\langle p_{\tilde{\tau}}\right\rangle=192.4 \pm 0.2 \mathrm{GeV}$ leads to a precise $\tilde{\tau}$ mass of

$$
m_{\tilde{\tau}}=157.6 \pm 0.2 \mathrm{GeV}
$$

The stau lifetime measurement is based on the decays of $\tilde{\tau}^{\prime} s$ which have been stopped in the detector. Requiring an isolated energetic cluster above a threshold of $10 \mathrm{GeV}$ or a muon above $10 \mathrm{GeV}$ originating somewhere inside the sensitive fiducial volume of the calorimeter or yoke, results in the decay time distribution shown in figure $2 \mathrm{c}$. A fit to the spectrum gives a $\tilde{\tau}$ lifetime of

$$
t_{\tilde{\tau}}=(2.6 \pm 0.05) \cdot 10^{6} \mathrm{~s},
$$

corresponding to roughly one month. The relation between stau lifetime and gravitino mass as a function of stau mass is graphically presented in figure $2 \mathrm{~d}$. For the present condition the lifetime increases as $t_{\tilde{\tau}} \sim m_{\tilde{G}}^{2}$ and rises significantly faster for $m_{\tilde{G}} \gtrsim 0.25 m_{\tilde{\tau}}$, reaching $10^{8} \mathrm{~s}$ for a $75 \mathrm{GeV}$ gravitino. Comparing with other physics scenarios one also observes that the lifetime can get very long for light $\tilde{\tau}^{\prime} s$ and small mass differences and, for a fixed gravitino mass, becomes shorter the heavier the stau.

A direct gravitino mass measurement can be performed by exploiting the $\tau$ recoil of the decay $\tilde{\tau} \rightarrow \tau \tilde{G}$, see eq. (6) and discussion in sect. 4. The upper endpoints of the energy spectra which coincide with the primary $\tau$ energy $E_{\tau}=77.5 \mathrm{GeV}$, are directly related to the masses involved. The leptonic 3-body decay $\tau \rightarrow e \nu \nu$ is not very useful due to the soft spectrum peaking at low values. The hadronic 2-body decay $\tau \rightarrow \pi \nu$ produces a flat spectrum, where the upper edge is, however, strongly diluted due to resolution effects and limited statistics. Well defined upper edges are provided by the hadronic decays to heavier final states $\tau \rightarrow \rho \nu$ and $\tau \rightarrow \pi \pi \pi \nu$. The energy distribution of both decay modes, defined as ' $\tau$ jets', is shown in figure $2 \mathrm{~b}$. In order to illustrate the sensitivity to the gravitino mass simulations assuming the nominal value of $m_{\tilde{G}}=20 \mathrm{GeV}$ and values shifted 

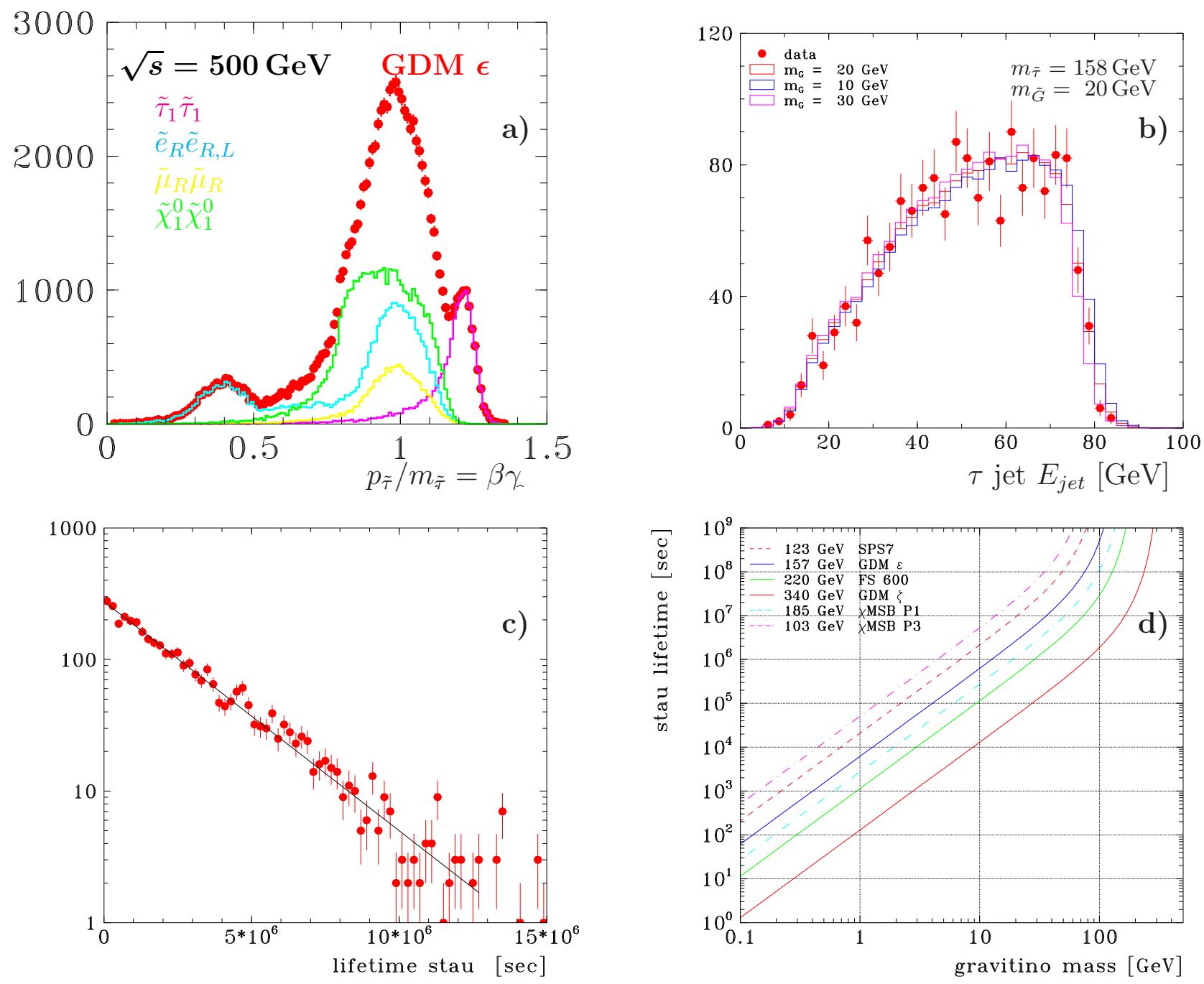

Figure 2: a) Scaled momentum spectrum $p / m=\beta \gamma$ of $\tilde{\tau}$ production with contributions from $\tilde{\tau}_{1} \tilde{\tau}_{1}$ (magenta), $\tilde{e}_{R} \tilde{e}_{R, L}$ (cyan), $\tilde{\mu}_{R} \tilde{\mu}_{R}$ (yellow) and $\tilde{\chi}_{1}^{0} \tilde{\chi}_{1}^{0}$ (green); b) $\tau$ recoil spectrum of the decay $\tilde{\tau}_{1} \rightarrow \tau \tilde{G}$ with $E_{j e t}$ of $\tau \rightarrow \rho \nu_{\tau}+3 \pi \nu_{\tau}$ compared with simulations assuming $m_{\tilde{G}}=20 \mathrm{GeV}$ (red histogram), $10 \mathrm{GeV}$ (blue) and $30 \mathrm{GeV}$ (magenta); c) $\tilde{\tau}$ lifetime distribution; d) $\tilde{\tau}$ lifetime versus $\tilde{G}$ mass for various Susy scenarios representing different $\tilde{\tau}$ masses. Statistics correspond to $\mathrm{GDM} \epsilon$ scenario, $\mathcal{L}=100 \mathrm{fb}^{-1}$ at $\sqrt{s}=500 \mathrm{GeV}$

by $\pm 10 \mathrm{GeV}$ are shown as well. A fit to the 'jet' energy spectrum, using either an analytical formula for the endpoint or a complete simulation, yields a gravitino mass

$$
m_{\tilde{G}}=20 \pm 4 \mathrm{GeV} .
$$

Taking all results together one can test the gravitational coupling of the stau to the gravitino and access the Planck scale, respectively Newton's constant. Inserting the expected values and accuracies on $m_{\tilde{\tau}}, t_{\tilde{\tau}}$ and $m_{\tilde{G}}$ in eq. (3) one finds for the supergravity Planck scale

$$
M_{P}=(2.4 \pm 0.5) \cdot 10^{18} \mathrm{GeV},
$$

where the error is dominated by the precision on the gravitino mass measurement.

The gravitino mass can be deduced more precisely from the $\tilde{\tau}$ mass and lifetime, if the gravitational coupling is assumed and the macroscopic value of $M_{P}$ is used in the decay-width of eq. (2). The resulting gravitino mass is $m_{\tilde{G}}=20 \pm 0.2 \mathrm{GeV}$, where the error is dominated by the lifetime measurement. 
It is a unique feature of gravitino LSP scenarios that the Planck scale can be directly measured in microscopic particle experiments by studying the properties of the NLSP and its decay. A further interesting test to reveal the nature of the gravitino as the supersymmetric partner of the graviton would be to determine the spin. This is in principle possible by studying correlations in the radiative decay $\tilde{\tau} \rightarrow \tau \gamma \tilde{G}$ [3], see also sect. 6 . Experimentally this is quite challenging, because it requires to distinguish single photons from $\pi^{0}$ decays, and in addition the expected rates are lower by two orders of magnitude.

\section{2. mSUGRA scenario GDM $\zeta$}

Due to the heavy sleptons in model GDM $\zeta$ a higher centre of mass energy of $\sqrt{s}=800 \mathrm{GeV}$ and an integrated luminosity of $\mathcal{L}=1 \mathrm{ab}^{-1}$ is chosen. The $\beta \gamma$ distribution of the produced $\tilde{\tau}^{\prime} s$ (inclusive cross section $\sigma_{S U S Y}=5 \mathrm{fb}$ ) is shown in figure $3 \mathrm{a}$. It exhibits the typical behaviour of slepton production close to threshold: a pronounced peak at $\beta \gamma \simeq 0.6$ from $\tilde{\tau}_{1} \tilde{\tau}_{1}$ pairs and another enhancement around 0.3 from $\tilde{e}_{R}$ and $\tilde{\mu}_{R}$ cascade decays, resulting in 1350 (850) trapped $\tilde{\tau}^{\prime} s$ in the hadron calorimeter (yoke). The analyses of the momentum spectrum yields a $\tilde{\tau}$ mass of $m_{\tilde{\tau}_{1}}=340.2 \pm 0.2 \mathrm{GeV}$. From the decay time distribution one gets the lifetime $t_{\tilde{\tau}}=(1.8 \pm 0.06) \cdot 10^{6} \mathrm{~s}$. The $\tau$ jet energy spectrum of the $\tilde{\tau}$ decay, shown in figure $3 \mathrm{~b}$, extends over a wide range. The edge of the endpoint energy, being less pronounced than in the previous example, can be used to get a direct gravitino mass measurement of $m_{\tilde{G}}=100 \pm 10 \mathrm{GeV}$. This value may be compared with the gravitino mass $m_{\tilde{G}}=100 \pm 2 \mathrm{GeV}$ calculated from the stau mass and lifetime.

Note: If higher energies were accessible at the ILC, then data taking at $\sqrt{s}=900 \mathrm{GeV}$, i.e. just above $\tilde{\chi}_{1}^{0} \tilde{\chi}_{1}^{0}$ threshold, increases the number of trapped $\tilde{\tau}^{\prime} s$ by a factor of 1.5 in the GDM $\zeta$ scenario.


Figure 3: a) Scaled momentum spectrum $p / m=\beta \gamma$ of $\tilde{\tau}$ production with contributions from $\tilde{\tau}_{1} \tilde{\tau}_{1}$ (magenta), $\tilde{e}_{R} \tilde{e}_{R}$ (cyan) and $\tilde{\mu}_{R} \tilde{\mu}_{R}$ (yellow); b) $\tau$ recoil spectrum of the decay $\tilde{\tau}_{1} \rightarrow \tau \tilde{G}$ with $E_{\text {jet }}$ of $\tau \rightarrow \rho \nu_{\tau}+3 \pi \nu_{\tau}$ compared with simulations using $m_{\tilde{G}}=100 \mathrm{GeV}$ (red histogram), $80 \mathrm{GeV}$ (blue) and $120 \mathrm{GeV}$ (magenta). GDM $\zeta$ scenario, $\mathcal{L}=1000 \mathrm{fb}^{-1}$ at $\sqrt{s}=800 \mathrm{GeV}$

The GDM $\eta$ scenario has an almost identical sparticle spectrum and differs essentially only by the lower gravitino mass of $m_{\tilde{G}}=20 \mathrm{GeV}$. This causes a much shorter lifetime, but, more importantly, the $\tau$ recoil energy spectrum is not really sensitive to the gravitino mass $\left(m_{\tilde{G}} / m_{\tilde{\tau}}=0.06\right)$ and can only be used to set an upper limit. 


\section{3. mSUGRA scenario FS 600}

The model FS 600 is investigated at a cms energy $\sqrt{s}=500 \mathrm{GeV}$, close to threshold pair production of the moderately heavy sleptons and neutralinos. A larger integrated luminosity is needed in order to achieve similar accuracies as for the other mSUGRA benchmarks. The results of the analysis are given in table III. It is worth noting that operating the ILC at a slightly higher energy of $520 \mathrm{GeV}$ leads to higher cross sections and a significant increase by a factor of 1.5 for the rate of trapped $\tilde{\tau}^{\prime} s$.

\subsection{Gauge mediated symmetry breaking scenario SPS 7}

The SPS 7 scenario has a relatively light sparticle spectrum and is investigated assuming $\sqrt{s}=410 \mathrm{GeV}$ and $\mathcal{L}=100 \mathrm{fb}^{-1}$, with a large cross section of $\sigma_{S U S Y}=420 \mathrm{fb}$ for inclusive $\tilde{\tau}$ production. As can be seen in the $\beta \gamma$ distribution of figure $4 \mathrm{a}$, most $\tilde{\tau}^{\prime} s$ leave the detector. There is, however, a large signal at $\beta \gamma \simeq 0.4$ from $\tilde{e}_{R} \tilde{e}_{L}$ production just above threshold, which contributes to the sample of 10000 (4900) trapped $\tilde{\tau}^{\prime} s$ in the calorimeter (yoke). The analysis of the $\tilde{\tau}$ momentum spectrum yields $m_{\tilde{\tau}_{1}}=124.3 \pm 0.1 \mathrm{GeV}$ and from a fit to the decay time distribution one obtains $t_{\tilde{\tau}}=209.3 \pm 2.4 \mathrm{~s}$. These values can be used to derive a very accurate gravitino mass of $m_{\tilde{G}}=100 \pm 1 \mathrm{MeV}$ assuming a gravitational coupling, see figure $2 \mathrm{~d}$. The $\tau$ recoil energy spectrum, shown in figure $4 \mathrm{~b}$, is not sensitive to such low gravitino masses and can only serve to give an upper limit on a direct measurement of $m_{\tilde{G}}<9 \mathrm{GeV}$ at $95 \%$ confidence level.
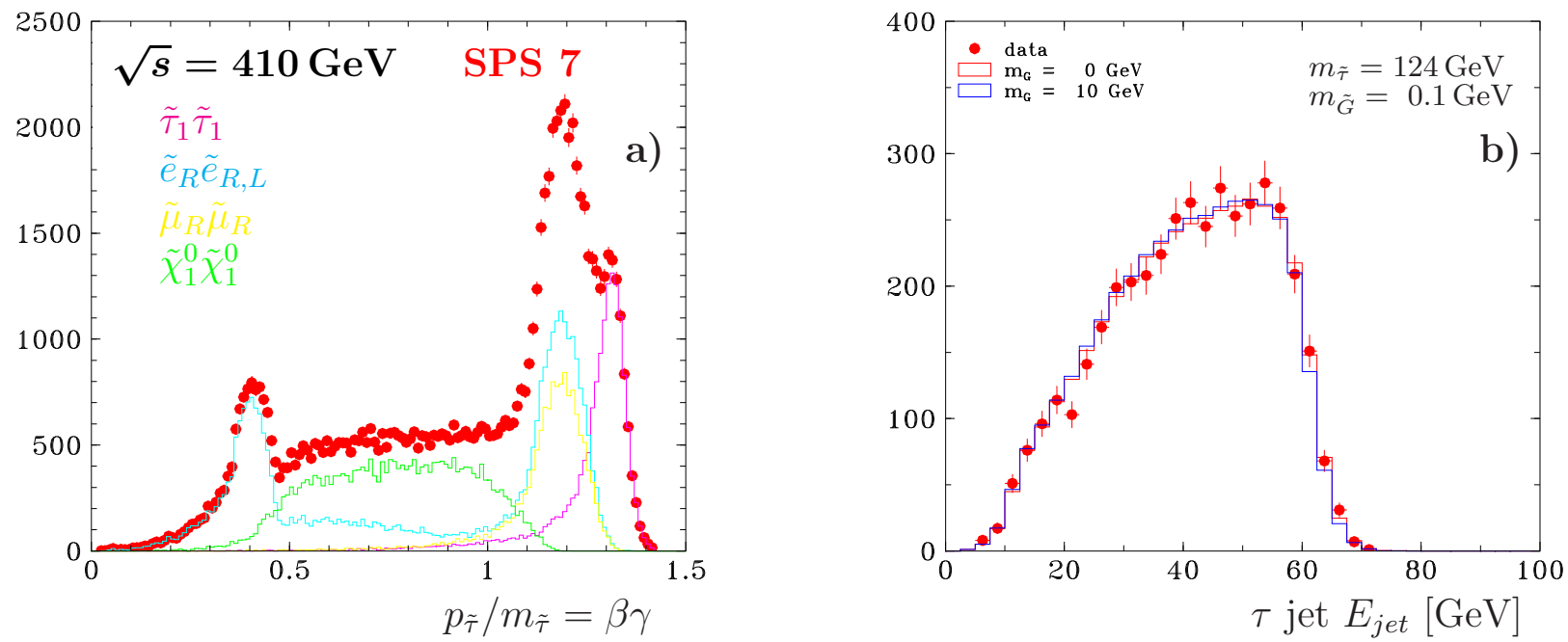

Figure 4: a) Scaled momentum spectrum $p / m=\beta \gamma$ of $\tilde{\tau}$ production with contributions from $\tilde{\tau}_{1} \tilde{\tau}_{1}$ (magenta), $\tilde{e}_{R} \tilde{e}_{R, L}$ (cyan), $\tilde{\mu}_{R} \tilde{\mu}_{R}$ (yellow) and $\tilde{\chi}_{1}^{0} \tilde{\chi}_{1}^{0}$ (green); b) $\tau$ recoil spectrum of the decay $\tilde{\tau}_{1} \rightarrow \tau \tilde{G}$ with $E_{j e t}$ of $\tau \rightarrow \rho \nu_{\tau}+3 \pi \nu_{\tau}$ compared with simulations using $m_{\tilde{G}}=0 \mathrm{GeV}$ (red histogram) and $10 \mathrm{GeV}$ (blue). SPS 7 scenario, $\mathcal{L}=100 \mathrm{fb}^{-1}$ at $\sqrt{s}=410 \mathrm{GeV}$

In the present case one does not profit from the calorimetric information to asses the gravitino mass directly and relies entirely on the stau properties. One may afford to run at higher energy, thereby shifting the $\beta \gamma$ spectrum of figure 4 a to larger values. For example, running the ILC at the 'canonical' energy $\sqrt{s}=500 \mathrm{GeV}$ reduces the $\tilde{\tau}_{1} \tilde{\tau}_{1}$ rate by $10 \%$ and the sample of trapped $\tilde{\tau}^{\prime} s$ by a factor of two, degrading the precision on the $\tilde{\tau}$ lifetime to $2 \%$.

Note: The relative precision on the $\tilde{\tau}$ lifetime measurement does not depend on the gravitino mass, should it be much lighter as often assumed in gauge mediated supersymmetry models. Technically, there may be a limitation to measure very short lifetimes below a millisecond. In the present method the $\tilde{\tau}$ decay is required to occur uncorrelated to any beam collision, which essentially means outside a time interval of $\Delta t=1 \mathrm{~ms}$ for 
a whole bunch train, repeating at a rate of $5 \mathrm{~Hz}$. As an illustration, in the SPS 7 scenario a $\tilde{\tau}$ lifetime of $5 \mathrm{~ms}$ corresponds to a gravitino mass of $0.5 \mathrm{MeV}$.

\subsection{Gaugino mediated symmetry breaking scenarios P3}

The model P3 is investigated at $\sqrt{s}=500 \mathrm{GeV}$ and assuming $\mathcal{L}=100 \mathrm{fb}^{-1}$. The inclusive $\tilde{\tau}$ cross section of $\sigma_{S U S Y}=470 \mathrm{fb}$ provides large rates, as can be seen in the $\beta \gamma$ spectrum of figure $5 \mathrm{a}$. The main contributions to the sample of stopped $\tilde{\tau}^{\prime} s$ come from $\tilde{\chi}_{1}^{0} \tilde{\chi}_{1}^{0}$ and $\tilde{e}_{R} \tilde{e}_{L}$ production, one expects about 3900 and 3700 particles in the calorimeter and yoke, respectively. From the kinematics of $\tilde{\tau}_{1} \tilde{\tau}_{1}$ production one gets a $\tilde{\tau}$ mass of $m_{\tilde{\tau}_{1}}=$ $102.5 \pm 0.2 \mathrm{GeV}$. A fit to the $\tilde{\tau}$ lifetime distribution yields $t_{\tilde{\tau}}=(4.2 \pm 0.1) \cdot 10^{7} \mathrm{~s}$. A direct measurement of the gravitino mass using the $\tau$ recoil energy spectrum of $\tilde{\tau} \rightarrow \tau \tilde{G}$, as displayed in figure $5 \mathrm{~b}$, provides a value of $m_{\tilde{G}}=25 \pm 1.5 \mathrm{GeV}$. Alternatively, one derives a gravitino mass of $m_{\tilde{G}}=25 \pm 0.3 \mathrm{GeV}$ from the $\tilde{\tau}$ lifetime measurement.

Note: The number of trapped $\tilde{\tau}^{\prime} s$ in the calorimeter can be increased by a factor of 1.8 when running at a slightly lower cms energy $\sqrt{s}=480 \mathrm{GeV}$.
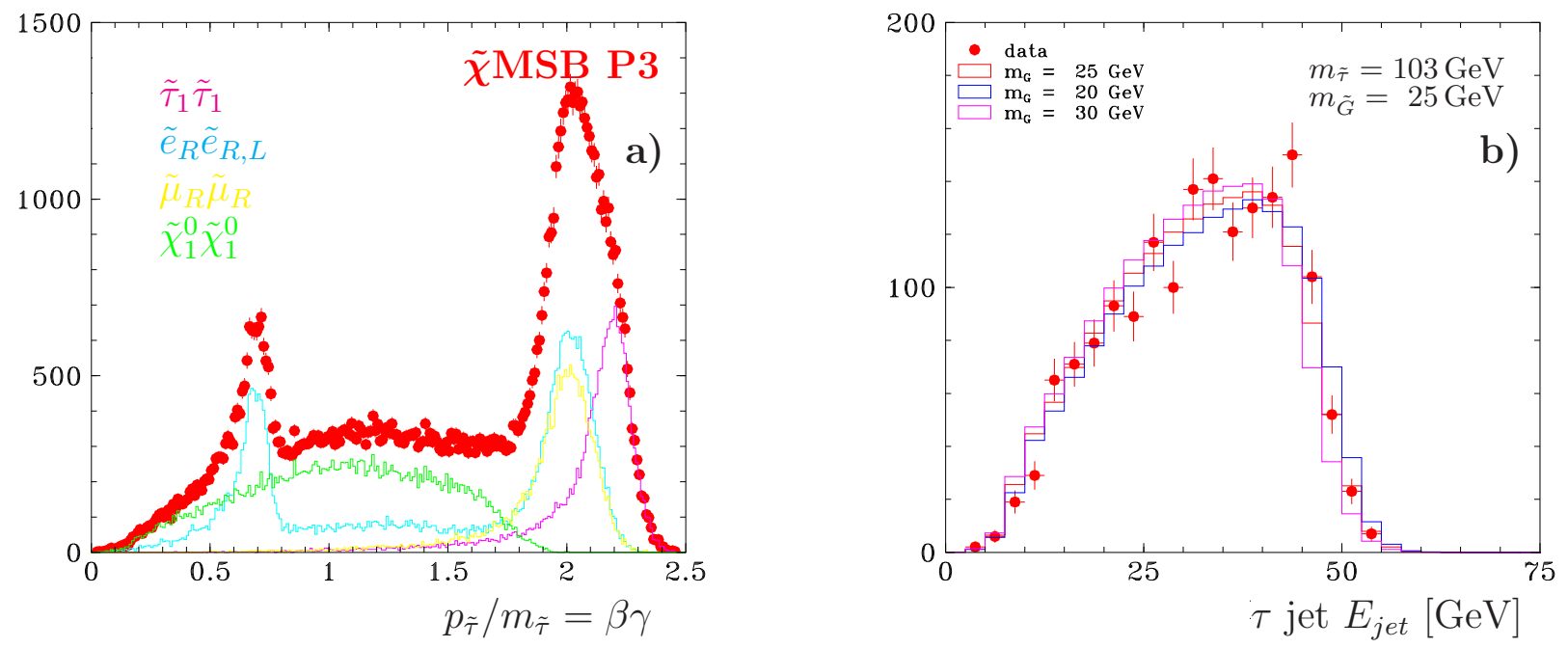

Figure 5: a) Scaled momentum spectrum $p / m=\beta \gamma$ of $\tilde{\tau}$ production with contributions from $\tilde{\tau}_{1} \tilde{\tau}_{1}$ (magenta), $\tilde{e}_{R} \tilde{e}_{R, L}$ (cyan), $\tilde{\mu}_{R} \tilde{\mu}_{R}$ (yellow) and $\tilde{\chi}_{1}^{0} \tilde{\chi}_{1}^{0}$ (green); b) $\tau$ recoil spectra of the decay $\tilde{\tau}_{1} \rightarrow \tau \tilde{G}$ with $E_{\text {jet }}$ of $\tau \rightarrow \rho \nu_{\tau}+3 \pi \nu_{\tau}$ compared with simulations assuming $m_{\tilde{G}}=25 \mathrm{GeV}$ (red histogram), $20 \mathrm{GeV}$ (blue) and $30 \mathrm{GeV}$ (magenta). $\tilde{\chi} \mathrm{MSB}$ P3 scenario, $\mathcal{L}=100 \mathrm{fb}^{-1}$ at $\sqrt{s}=500 \mathrm{GeV}$

The scenario P3 yields a lifetime of about 1.3 years. Taking instead a larger gravitino mass of $m_{\tilde{G}}=50 \mathrm{GeV}$, as proposed by the authors of [14], the lifetime increases by almost an order of magnitude, and it may be difficult to observe all accumulated $\tilde{\tau}^{\prime} s$ during the operation of an experiment. Assuming a measurement period of three years will provide a lifetime of $t_{\tilde{\tau}}=(3.9 \pm 0.7) \cdot 10^{8} \mathrm{~s}$, corresponding to 12.4 years. The relative error grows faster than expected from the reduced statistics (factor 4 less decays) when observing only a fraction of the lifetime. Of coarse, this has also consequences for the measurement of the decay spectra.

The $\tilde{\chi} M S B P 1$ scenario is characterised by somewhat heavier sparticles and is investigated at $\sqrt{s}=420 \mathrm{GeV}$, just above threshold production of sleptons and the lightest neutralino. Again, the event statistics allow measurements to be performed at the per mill to per cent level, see table III. 


\section{Discussion of results}

If kinematically accessible, metastable staus are copiously produced at the ILC. They are easy to detect and their properties, like mass and lifetime, and the decays into gravitinos can be studied in great detail. The results of the various case studies of the supersymmetry models and the assumed experimental conditions are summarised in table III. The common features of all spectra concerning the achievable accuracies of the basic measurements are: (i) the $\tilde{\tau}$ mass can be measured at the per mill level by exploiting the 2-body kinematics of $\tilde{\tau}_{1} \tilde{\tau}_{1}$ pair production in the tracking detector; (ii) the $\tilde{\tau}$ lifetime can be determined within a few per cent by observing the decays $\tilde{\tau} \rightarrow \tau \tilde{G}$ triggered in the calorimeter and/or iron yoke; and (iii) the $\tilde{G}$ mass can be accessed directly with a precision of order $10 \%$ (degrading rapidly for $m_{\tilde{G}} / m_{\tilde{\tau}} \lesssim 0.1$ ) by measuring the $\tau$ recoil spectra of the $\tilde{\tau}$ decays in the calorimeter.

Table III: Expected accuracies on the determination of $\tilde{\tau}$ and $\tilde{G}$ properties for various SusY scenarios: stau mass $m_{\tilde{\tau}}$, stau lifetime $t_{\tilde{\tau}}$, gravitino mass $m_{\tilde{G}}^{E_{\tau}}$ from $\tau$ recoil energy spectra and gravitino mass $m_{\tilde{\tilde{G}}}^{t_{\tilde{\tau}}}$ deduced from $\tilde{\tau}$ lifetime. Experimental conditions in the last columns: centre of mass energy $\sqrt{s}$, inclusive $\tilde{\tau}$ production cross section $\sigma_{\tilde{\tau} \tilde{\tau} X}$, integrated luminosity $\mathcal{L}$, number of trapped $\tilde{\tau}^{\prime} s$ in hadron calorimeter $N_{\tilde{\tau}}^{\text {hcal }}$ and instrumented yoke $N_{\tilde{\tau}}^{\text {yoke }}$

\begin{tabular}{|c|c|c|c|c|c|c|c|c|c|}
\hline & $m_{\tilde{\tau}}[\mathrm{GeV}]$ & $t_{\tilde{\tau}}[\mathrm{s}]$ & $m_{\tilde{G}}^{E_{\tau}}[\mathrm{GeV}]$ & $m_{\tilde{G}}^{t_{\tilde{\tau}}}[\mathrm{GeV}]$ & $\sqrt{s}[\mathrm{GeV}]$ & $\sigma_{\tilde{\tau} \tilde{\tau} X}[\mathrm{fb}]$ & $\mathcal{L}\left[\mathrm{fb}^{-1}\right]$ & $N_{\tilde{\tau}}^{\text {hcal }}$ & $N_{\tilde{\tau}}^{\text {yoke }}$ \\
\hline 1 SPS 7 & $124.3 \pm 0.1$ & $209.3 \pm 2.4$ & $<9$ & $0.1 \pm 0.001$ & 410 & 420 & 100 & 10000 & 4900 \\
\hline 2 FS 600 & $219.3 \pm 0.2$ & $(3.6 \pm 0.1) 10^{6}$ & $50 \pm 9$ & $50 \pm 0.7$ & 500 & 20 & 250 & 2100 & 4200 \\
\hline $3 \mathrm{GDM} \epsilon$ & $157.6 \pm 0.2$ & $(2.6 \pm 0.05) 10^{6}$ & $20 \pm 4$ & $20 \pm 0.2$ & 500 & 300 & 100 & 4100 & 1850 \\
\hline $4 \mathrm{GDM} \zeta$ & $340.2 \pm 0.2$ & $(1.8 \pm 0.06) 10^{6}$ & $100 \pm 10$ & $100 \pm 2$ & 800 & 5 & 1000 & 1350 & 800 \\
\hline 5 GDM $\eta$ & $322.1 \pm 0.2$ & $(6.9 \pm 0.3) 10^{4}$ & $20 \pm 25$ & $20 \pm 0.4$ & 800 & 9 & 1000 & 1050 & 1850 \\
\hline $6 \tilde{\chi} \mathrm{MSB} \mathrm{P} 1$ & $185.2 \pm 0.1$ & $(9.1 \pm 0.2) 10^{6}$ & $50 \pm 3$ & $50 \pm 0.6$ & 420 & 27 & 200 & 3700 & 4100 \\
\hline $7 \tilde{\chi} \mathrm{MSB}$ P3 & $102.5 \pm 0.2$ & $(4.2 \pm 0.1) 10^{7}$ & $25 \pm 1.5$ & $25 \pm 0.3$ & 500 & 470 & 100 & 3900 & 3700 \\
\hline
\end{tabular}

These expectations are based on moderate integrated luminosities, the event rates to be accumulated during one to three years of data taking at the ILC under nominal conditions. Although the $\tilde{\tau}$ decays are treated in a parametrised form the results should be reliable within a factor of two. A detailed detector simulation may lead to a reduction of the data samples due to acceptance cuts, quality criteria and resolution effects. However, the losses may be compensated and the event statistics can be considerably increased by optimising the beam energies (see sect. 5) and making use of the beam polarisations. For instance, the production cross sections for light sleptons, $\tilde{\tau}_{1} \tilde{\tau}_{1}, \tilde{e}_{R} \tilde{e}_{R}$ and $\tilde{\mu}_{R} \tilde{\mu}_{R}$, are enhanced by a factor $\left(1+\mathcal{P}_{e^{-}}\right)\left(1-\mathcal{P}_{e^{+}}\right) \simeq 2.9$ when using right-handed polarised electrons of degree $\mathcal{P}_{e^{-}}=+0.8$ and left-handed polarised positrons of $\mathcal{P}_{e^{+}}=-0.6$.

Concerning the expected ILC detector performance with respect to detecting metastable $\tilde{\tau}^{\prime} s$ there is, however, a caveat. At present, it is envisaged to operate the LDC calorimeters in a pulsed mode [19]. In order to cope with the heat production of the electronics, it is foreseen that the amplifiers and read out channels of the detector parts will be switched on and read out just during one bunch train for 2 ms, and then be switched off to wait for the next bunch train occurring $200 \mathrm{~ms}$ later; i.e. the detector is inactive for most of the time. Such an operation is designed for usual, beam correlated physics scenarios, but clearly has to be revised if one wants to observe the decays of metastable particles. For the iron yoke instrumentation it does not really seem to be necessary to apply a power pulsing. A quasi-continuous read-out of all channels above a certain threshold over the so far 'idle' time gap of $198 \mathrm{~ms}$ appears possible, replacing the $\tilde{\tau}$ decay trigger by software analysis [26]. Thus a lifetime measurement will be feasible in any case, may be at the expense of a lower event rate restricted to $\tilde{\tau}^{\prime} s$ trapped in the yoke. The goal to also increase the duty cycle of the hadron calorimeter substantially has to be pursued in future R\&D detector developments. Since a direct measurement of the gravitino mass is extremely important, it will be assumed in the following discussion that the technical problems will be solved and the calorimeter will be permanently sensitive with essentially no dead-time. 
An independent measurement of the gravitino mass is of prime importance. Applying eq. (3) one can determine the supergravity Planck scale from the NLSP decay with an accuracy of order $10 \%$. A value consistent with the macroscopic scale of gravity, i.e. Newton's constant $G_{N}=6.7 \cdot 10^{-39} \mathrm{GeV}^{-2}$, would be a decisive test that the gravitino is indeed the superpartner of the graviton. At the same time the measurement of the gravitino mass can be used to yield the supersymmetry breaking scale $F=\sqrt{3} M_{P} m_{3 / 2}$, see eq. (1), which is an important parameter to unravel the nature of the supersymmetry breaking mechanism.

From the $\tilde{\tau}$ mass and lifetime measurements one can compute the gravitino mass assuming that the $\tilde{\tau}$ decay occurs with gravitational coupling according to eq. (2). The expected accuracy is again of order per cent. This interpretation may be plausible, however, it can only be justified, if there is additional information which confirms the gravitino LSP assignment.

For too light gravitinos a direct mass measurement may not be possible, leaving the nature of the LSP open. An alternative option may be a spin $1 / 2$ axino $\tilde{a}$ as lightest supersymmetric particle [27]. In such a scenario the $\tilde{\tau}$ lifetime is not related to the axino mass and, depending on the parameters, may vary between some $0.01 \mathrm{sec}$ and 10 hours, comparable to the expectations of gauge mediated and gravity mediated SusY breaking models. A method to differentiate between these two scenarios is to study the radiative three-body decays $\tilde{\tau} \rightarrow \tau \gamma \tilde{G}$ and $\tilde{\tau} \rightarrow \tau \gamma \tilde{a}$. The branching ratios are suppressed by two orders of magnitude and expected to be slightly larger for the axino LSP, the exact values depend on the selected phase space to identify an isolated photon. More promising is to investigate $\gamma-\tau$ correlations of the decay topology. In the case of gravitino LSP the photons are preferentially emitted collinear with the $\tau$ and have a soft, Bremsstrahlung like energy spectrum. A similar configuration also occurs for the axino LSP, however, there is in addition a substantial rate of energetic photons, $E_{\gamma} \rightarrow m_{\tilde{\tau}} / 2$, emitted opposite, back-to-back to the $\tau$ direction. Such a signature would clearly allow to distinguish between the interpretations as axino LSP or as gravitino LSP. Experimentally the analysis of radiative $\tilde{\tau}$ decays is quite ambitious because one has to discriminate a single photon against the photons from $\pi^{0}$ decays as well as against the hadrons in hadronic $\tau$ decays. The expected low event rates will be further reduced by efficient selection criteria. Hence, large statistics data samples are required, which may be provided by the ILC.

\section{Conclusions}

It has been shown in detailed analyses that future ILC experiments have a rich potential to study SUSY scenarios where the gravitino $\tilde{G}$ is the lightest supersymmetric particle and a charged stau $\tilde{\tau}$ is the long-lived, metastable next-to-lightest supersymmetric particle. Precise determinations of the $\tilde{\tau}$ mass and lifetime and

of the $\tilde{G}$ mass appear feasible, provided the proposed detectors will operate at a reasonable duty cycle. A calorimetric measurement of the gravitino mass from the $\tau$ recoil spectra of the decay $\tilde{\tau} \rightarrow \tau \tilde{G}$ gives access to the gravitational coupling, i.e. to the Planck scale, in a microscopic particle experiment and thus provides a unique test of supergravity. Furthermore these observations will put stringent constraints on an interpretation of the gravitino as dark matter candidate, being undetectable in astrophysical search experiments, and will allow its relic density to be to computed reliably.

Acknowledgements. I want to thank W. Buchmüller and P. M. Zerwas for many valuable discussions and comments on the manuscript.

\section{References}

[1] H. Pagels and J. R. Primack, Phys. Rev. Lett. 48 (1982) 223; M. Y. Khlopov and A. D. Linde, Phys. Lett. B 138 (1984) 265; J. R. Ellis, J. E. Kim and D. V. Nanopoulos, Phys. Lett. B 145 (1984) 181; M. Bolz, W. Buchmüller and M. Plümacher, Phys. Lett. B 443 (1998) 209 [arXiv:hep-ph/9809381]. 
[2] S. Eidelman et al. [Particle Data Group], Phys. Lett. B 592 (2004) 1.

[3] W. Buchmüller, K. Hamaguchi, M. Ratz and T. Yanagida, Phys. Lett. B 588 (2004) 90 [arXiv:hep$\mathrm{ph} / 0402179]$.

[4] M. Bolz, A. Brandenburg and W. Büchmuller, "Thermal production of gravitinos," Nucl. Phys. B 606 (2001) 518 [arXiv:hep-ph/0012052].

[5] J. L. Feng, A. Rajaraman and F. Takayama, Phys. Rev. Lett. 91 (2003) 011302 [arXiv:hep-ph/0302215] and Phys. Rev. D 68 (2003) 063504 [arXiv:hep-ph/0306024].

[6] K. Hamaguchi, Y. Kuno, T. Nakaya and M. M. Nojiri, Phys. Rev. D 70 (2004) 115007 [arXiv:hep$\mathrm{ph} / 0409248]$.

[7] J. L. Feng and B. T. Smith, Phys. Rev. D 71 (2005) 015004 [Erratum-ibid. D 71 (2005) 0109904] [arXiv:hep$\mathrm{ph} / 0409278]$.

[8] A. De Roeck, J. R. Ellis, F. Gianotti, F. Moortgat, K. A. Olive and L. Pape, arXiv:hep-ph/0508198.

[9] M. Dine, A. E. Nelson and Y. Shirman, Phys. Rev. D 51 (1995) 1362 [arXiv:hep-ph/9408384].

[10] T. Moroi, H. Murayama and M. Yamaguchi, Phys. Lett. B 303 (1993) 289.

[11] B.C. Allanach et al., Eur. Phys. J. C 25 (2002) 113 [arXiv:hep-ph/0202233].

[12] H. P. Nilles, Phys. Rept. 110 (1984) 1.

[13] D. E. Kaplan, G. D. Kribs and M. Schmaltz, Phys. Rev. D 62 (2000) 035010 [arXiv:hep-ph/9911293];

Z. Chacko, M. A. Luty, A. E. Nelson and E. Ponton, JHEP 0001 (2000) 003 [arXiv:hep-ph/9911323].

[14] W. Buchmüller, J. Kersten and K. Schmidt-Hoberg, JHEP 0602 (2006) 069 [arXiv:hep-ph/0512152].

[15] W. Buchmüller, K. Hamaguchi and J. Kersten, Phys. Lett. B 632 (2006) 366 [arXiv:hep-ph/0506105].

[16] W. Porod, Comput. Phys. Commun. 153 (2003) 275-315 [arXiv:hep-ph/0301101].

[17] A. Djouadi, J. L. Kneur and G. Moultaka, arXiv:hep-ph/0211331.

[18] Tesla Technical Design Report, DESY 2001-011, Part IV: A Detector for TESLA.

[19] Large Detector Concept working group, http://www.ilcdc.org

[20] B Rossi, 'High-Energy Particles', Prentice-Hall, Inc (1952).

[21] T. Sjöstrand, P. Edén, C. Friberg, L. Lönnblad, G. Miu, S. Mrenna and E. Norrbin, Comput. Phys. Commun. 135 (2001) 238 [arXiv:hep-ph/0010017].

[22] T. Ohl, Comput. Phys. Commun. 101 (1997) 269 [arXiv:hep-ph/9607454].

[23] M. Pohl and H. J. Schreiber, DESY-02-061, arXiv:hep-ex/0206009.

[24] A. Freitas, A. von Manteuffel and P. M. Zerwas, Eur. Phys. J. C 34 (2004) 487 [arXiv:hep-ph/0310182], and Eur. Phys. J. C 40 (2005) 435 [arXiv:hep-ph/0408341]; A. Freitas, D. J. Miller and P. M. Zerwas, Eur. Phys. J. C 21 (2001) 361 [arXiv:hep-ph/0106198].

[25] J. L. Feng and M. E. Peskin, Phys. Rev. D 64 (2001) 115002 [arXiv:hep-ph/0105100].

[26] G. Eckerlin, private communication.

[27] A. Brandenburg, L. Covi, K. Hamaguchi, L. Roszkowski and F. D. Steffen, Phys. Lett. B 617 (2005) 99 [arXiv:hep-ph/0501287]. 